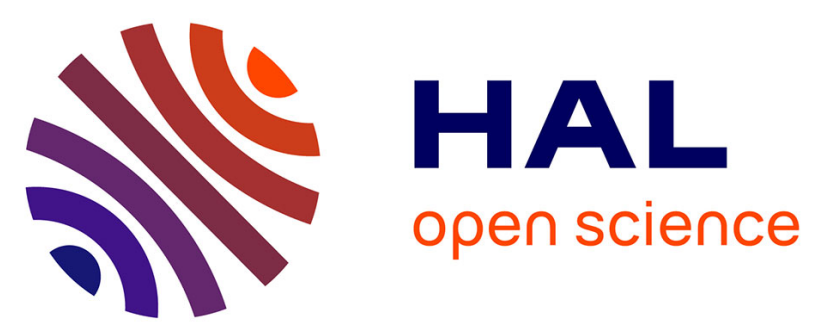

\title{
Modular Polyphased Full Order Current State-Space Model of the Modular Multilevel Converter
}

\author{
Grégoire Le Goff, Maurice Fadel, Marc Bodson
}

\section{To cite this version:}

Grégoire Le Goff, Maurice Fadel, Marc Bodson. Modular Polyphased Full Order Current StateSpace Model of the Modular Multilevel Converter. 2021 IEEE 19th International Power Electronics and Motion Control Conference (PEMC), Apr 2021, Gliwice (on line), Poland. pp.132-139, 10.1109/PEMC48073.2021.9432502 . hal-03349291

\section{HAL Id: hal-03349291 \\ https://hal.science/hal-03349291}

Submitted on 20 Sep 2021

HAL is a multi-disciplinary open access archive for the deposit and dissemination of scientific research documents, whether they are published or not. The documents may come from teaching and research institutions in France or abroad, or from public or private research centers.
L'archive ouverte pluridisciplinaire HAL, est destinée au dépôt et à la diffusion de documents scientifiques de niveau recherche, publiés ou non, émanant des établissements d'enseignement et de recherche français ou étrangers, des laboratoires publics ou privés. 


\title{
Modular Polyphased Full Order Current State-Space Model of the Modular Multilevel Converter
}

\author{
Grégoire Le Goff \\ Maurice Fadel \\ LAPLACE \\ Marc Bodson \\ LAPLACE \\ Université de Toulouse, CNRS, INPT, UPS Université de Toulouse, CNRS, INPT, UPS \\ Toulouse, France \\ Toulouse, France \\ legoff@laplace.univ-tlse.fr \\ fadel@laplace.univ-tlse.fr \\ Electrical and Computer Engineering \\ University of Utah \\ Salt Lake City, Utah, USA \\ bodson@eng.utah.edu
}

\begin{abstract}
This paper presents a new state-space model (SSM) to represent the four types of currents flowing through a Modular Multilevel Converter (MMC). In line with the converter topology, the model itself is modular. In other words, its configuration adapts itself to the number of $\mathrm{AC}$ phases, to the active or passive nature of the $\mathrm{AC}$-side grid, to the connection between the $A C$ and DC neutral points, and to the number of submodules. The model describes the influence of the MMC arm voltages on the currents using an average behavior of the submodules. Simulations performed using a software dedicated to electrical engineering systems demonstrate the value and the accuracy of the proposed model.
\end{abstract}

Index Terms-MMC, State-Space Model, Polyphased System, Modular Model, Current Model

\section{INTRODUCTION}

\section{A. Modular Multilevel Converters}

Multilevel converters have significant advantages over classical converters for high power applications. They make it possible to lower the stress on the switching cells as well as the total harmonic distortion [1]. The modular multilevel converter (MMC) has a topology that is made of identical series-connected submodules (SM) [2]. The possibility of adding submodules gives scalability to the MMC for highpower and high-voltage applications. Therefore, the converter has enabled an acceleration of the deployment of HVDC transmission systems [3]. The more SMs an MMC has, the more degrees of freedom are available to control the power flowing trough the MMC.

\section{B. Orientation of the research work}

This paper contributes to an overall research objective aimed to develop control allocation methods for the MMC. The principle of this control method is to take advantage of the large number of redundant control variables to operate the system optimally [4]. In the case of the MMC, several SM connection configurations are possible to achieve the same level of voltage in an arm. Control allocation thus seems particularly suitable if a state-space model (SSM) can be developed that makes it possible to fully exploit this redundancy. In the objective to generalize the method, the model should also be modular, reflecting the nature of the converter that it represents.

The MMC is characterized by the quantity $N$ of SMs present in each of its arms and by the number $m$ of phases in

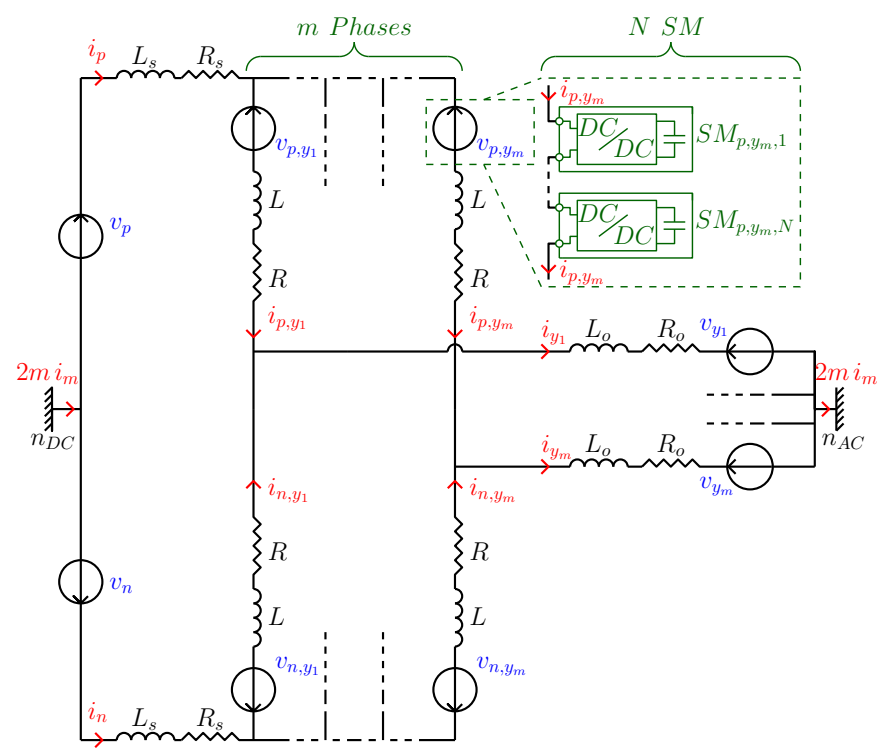

Fig. 1. Electrical diagram of the MMC with $m$ phases and $N$ SM per arm

the AC network to which it is connected, as shown in Fig.1. The model is thus modular according to these two quantities in order to allow the modeling, simulation and control of any MMC.

\section{Assumptions about the submodules}

Each SM is itself a DC-DC converter. There is a diversity of submodule topologies [5], but the principle remains the same: each SM contains a voltage source in the form of a capacitor (or capacitors) able to impose a voltage level within the arm to which the SM belongs. The imposed voltage level can be modulated by acting on the switches of the SM to connect it to the arm [6]. The half-bridge (chopper cell) and the full-bridge cell [5] are the main topologies used in the SMs composing most MMCs. In these two cases, the equilibrium voltage of a capacitor is $V_{D C} / N$, with $V_{D C}=v_{p}-v_{n}$ the DC bus voltage, [6]. Note that the subscript $p$ refers to the positive pole of the DC bus and the subscript $n$ to the negative one.

Since each SM contains a capacitor, the impedance encountered by the current flowing through an arm depends on the capacitance $C$ of the capacitors. In the approach used here, 
the choice is made to work with an average model for the SMs. Thus, the SMs of an entire arm are modeled by the equivalent capacitance $C^{e q}=C / N$ resulting from the series connection of the $N$ SMs, as is done in [7], [8] using the principle of average model detailed in [9]. In this approach, capacitor balancing is assumed to be ensured independently. As a consequence, the set of SMs of an arm is replaced by a variable voltage source, as shown in [10]-[12]. The representation is shown on Fig. 1 as $v_{x, y}(x \in\{p, n\}$ and $\left.y \in\left\{y_{1}, \ldots, y_{m}\right\}\right)$. Note that $y_{k}$ refers to $k$-th phase on ACside.

\section{Novelty of the proposed model}

From a high-level point-of-view, there are at most four types of currents that can appear during the operation of the MMC: the DC source current $\underline{I_{s}}$, the AC output currents $I_{o}$, the circulating currents between the arms of the MMC $I_{c}$, and the common mode current $I_{m}$ when the AC grid and the DC bus neutral points are connected. The behavior of these different currents is determined by the voltage levels in each arm of the MMC $\left(v_{x, y}, x \in\{p, n\}\right.$ and $\left.y \in\left\{y_{1}, \ldots, y_{m}\right\}\right)$, the DC bus voltage (represented by $v_{p}$ and $\left.v_{n}\right)$, and the AC voltages $\left(v_{y_{1}}\right.$ to $\left.v_{y_{m}}\right)$.

Previous works have decoupled the dynamics of these different current types for three-phase electrical systems like in [10], [13] where a matrix model is derived. A first novelty of the present paper is, taking inspiration from these studies and [14], to derive a non-matrix decoupled model of the currents extended to the $m$-phase case. The approaches of [7], [11], [14] shape a state-space model representing the dynamics of only three out of the four possible current types. Compared to [14], the paper presents a more limited, but also more explicit derivation of the converter model, in addition to considering the presence of a common mode current. The novelty of the current developed state-space model is to take into consideration all four possible current types, the possible active nature of the $\mathrm{AC}$-side grid and the possible connection of the AC and DC neutrals, as well as extending it to the $m$ phase case. To put it in a nutshell, the interest of the model introduced here is thus to have a state-space model which readily adapts to $m$ and $N$ without loss of generality on the possibilities of the DC bus, the AC network and the connection between the two.

\section{E. Outline}

Throughout the article, attention will be focused on the high-level model of the MMC. In a first step, the decoupled current model will be developed, from which the full order state-space model of the currents will be derived. The proposed model will then be tested in a parallel simulation using the PLECS ${ }^{1}$ software with MATLAB ${ }^{1}-$ Simulink $\AA$. As PLECS $\AA$ is a simulation software that embeds a detailed description of electrical components, its accuracy has been

\footnotetext{
${ }^{1}$ PLECS (Piecewise Linear Electrical Circuit Simulation) is a circuit-based simulation software for systems in electrical engineering.
}

proven. It will be considered as the reference allowing us to evaluate the accuracy of the introduced model.

\section{Current State-Space Model}

\section{A. Coupled Current Dynamics}

In order to explain the polyphase model, Fig. 2 focuses on a given leg, and thus phase, of the MMC structure. To simplify the notation, any time variable $x(t)$ will be denoted $x$ and the derivative operator is replaced by the LAPLACE variable, $s$. Applying the Kirchhoff voltage law on the current path flowing through the positive pole of the DC bus gives the following equation:

$$
\begin{aligned}
& \forall y \in\left\{y_{1}, \ldots, y_{m}\right\}: \\
& \left\{\begin{array}{c}
v_{p}=v_{y}+\left(R_{o}+L_{o} s\right) i_{y}+(R+L s) i_{p, y} \\
+v_{p, y}+\left(R_{s}+L_{s} s\right) i_{p}+v_{n_{A D}}
\end{array}\right.
\end{aligned}
$$

Where $v_{n_{A D}}=v_{n_{A C}}-v_{n_{D C}}$ is the voltage between the DCside and AC-side neutral points. Applying the same voltage law on the path linking to the negative pole of the DC bus gives:

$$
\begin{aligned}
& \forall y \in\left\{y_{1}, \ldots, y_{m}\right\}: \\
& \left\{\begin{array}{c}
v_{n}=v_{y}+\left(R_{o}+L_{o} s\right) i_{y}+(R+L s) i_{n, y} \\
+v_{n, y}+\left(R_{s}+L_{s} s\right) i_{n}+v_{n_{A D}}
\end{array}\right.
\end{aligned}
$$

Thus, (1) and (2) are summarized by the following equation to which the name $\left(\alpha_{x, y}\right)$ is given:

$$
\begin{aligned}
& \forall x \in\{p, n\}, \forall y \in\left\{y_{1}, \ldots, y_{m}\right\}: \\
& \left(\alpha_{x, y}\right):\left\{\begin{array}{c}
v_{y}+\left(R_{o}+L_{o} s\right) i_{y}+(R+L s) i_{x, y}+v_{x, y} \\
+\left(R_{s}+L_{s} s\right) i_{x}-v_{x}=-v_{n_{A D}}
\end{array}\right.
\end{aligned}
$$

Equation (3) brings to light the fact that the variable voltage sources, represented by $v_{x, y}$, control different types of current: $i_{s}, i_{y}$ and $i_{x, y}$. In other words, the behaviors of different current types are coupled. In the following, current decoupling will be addressed and the definition of the four types of current, previously mentionned in paragraph I-D, will be stated.

\section{B. Current Decomposition}

The currents flowing in the MMC can be split as shown in Fig. 3. Indeed, a first contribution will come from the common mode current $i_{m}$, a second from the DC bus $i_{s}$, a third from the currents flowing between the arms $i_{c_{y}}$ and a fourth from the AC side output $i_{o_{y}}$ [13]. According to the orientation convention of each type of current in the MMC legs, using the superposition of the four current types in each arm gives $\forall y \in\left\{y_{1}, \ldots, y_{m}\right\}$ :

$$
\begin{aligned}
& i_{p, y}=i_{m}+i_{s}+i_{c_{y}}+i_{o_{y}} \\
& i_{n, y}=i_{m}-i_{s}-i_{c_{y}}+i_{o_{y}}
\end{aligned}
$$

The sum and difference of (4) and (5) yield:

$$
\forall y \in\left\{y_{1}, \ldots, y_{m}\right\},\left\{\begin{array}{l}
i_{p, y}+i_{n, y}=2\left(i_{m}+i_{o_{y}}\right) \\
i_{p, y}-i_{n, y}=2\left(i_{s}+i_{c_{y}}\right)
\end{array}\right.
$$

From Fig. 3 (c) and (d), applying Kirchhoff's current law to the MMC structure implies that the sum of the circulating 


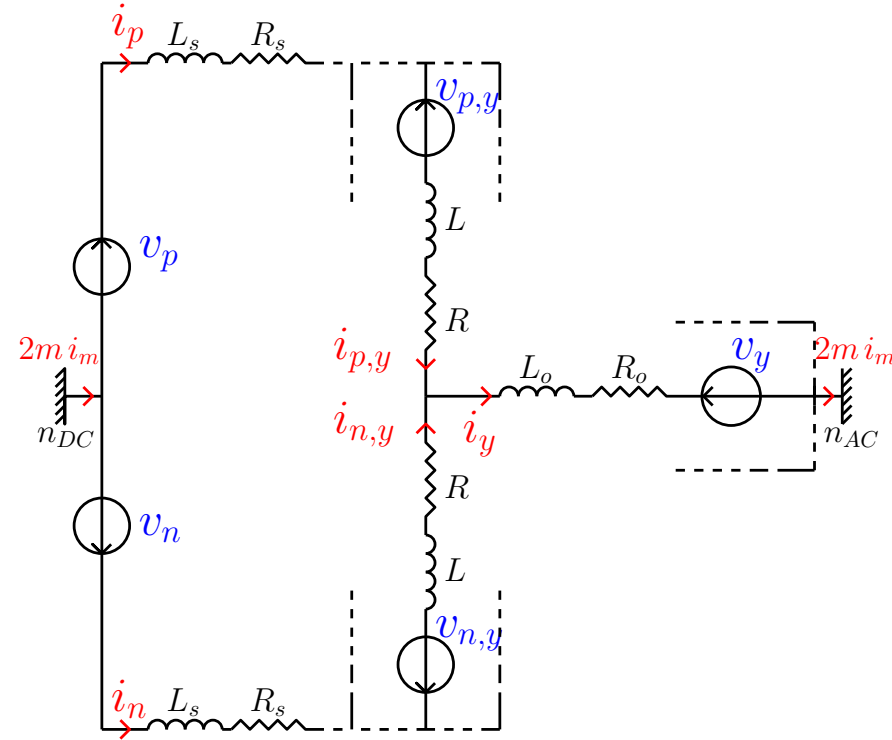

Fig. 2. Electrical diagram of the MMC's $y$-th arm

currents is null, as well as the sum of the output currents. Thus, $\sum_{y=y_{1}}^{y_{m}} i_{c_{y}}=0$ and $\sum_{y=y_{1}}^{y_{m}} i_{o_{y}}=0$, which, in turn, applied to the sum of (4) and (5) on all the legs, gives:

$$
\begin{aligned}
& i_{p}=\sum_{y=y_{1}}^{y_{m}} i_{p, y}=m\left(i_{m}+i_{s}\right) \\
& i_{n}=\sum_{y=y_{1}}^{y_{m}} i_{n, y}=m\left(i_{m}-i_{s}\right)
\end{aligned}
$$

Therefore, taking the sum and difference of (7) and (8) implies that:

$$
\begin{aligned}
& 2 m i_{m}=i_{p}+i_{n}=\sum_{y=y_{1}}^{y_{m}} i_{p, y}+i_{n, y} \\
& 2 m i_{s}=i_{p}-i_{n}=\sum_{y=y_{1}}^{y_{m}} i_{p, y}-i_{n, y}
\end{aligned}
$$

Fig. 2 brings another usefull equation. Indeed, the current flowing towards the AC-side is found, using the Kirchhoff's current law, to be:

$$
\forall y \in\left\{y_{1}, \ldots, y_{m}\right\}, i_{y}=i_{p, y}+i_{n, y}
$$

Substituting $i_{p, y}+i_{n, y}$ from (6), it follows that:

$$
\forall y \in\left\{y_{1}, \ldots, y_{m}\right\}, i_{y}=2\left(i_{m}+i_{o_{y}}\right)
$$

For the upcoming developments, consider the following expressions:

$\forall j \in \llbracket 1 ; m \rrbracket,\left\{\begin{array}{l}(m-1) i_{y_{j}}-\sum_{k \neq j}^{m} i_{y_{k}} \\ (m-1)\left(i_{p, y_{j}}-i_{n, y_{j}}\right)-\sum_{k \neq j}^{m}\left(i_{p, y_{k}}-i_{n, y_{k}}\right)\end{array}\right.$

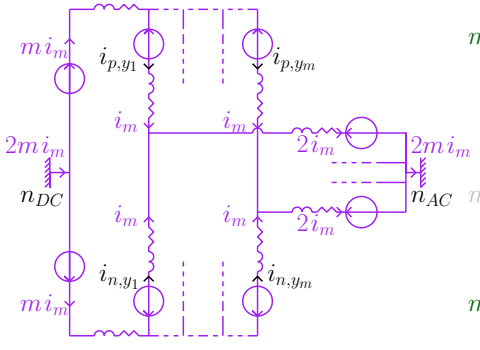

(a) Common Mode Current

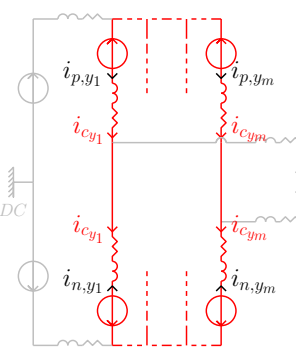

(c) Circulating Currents

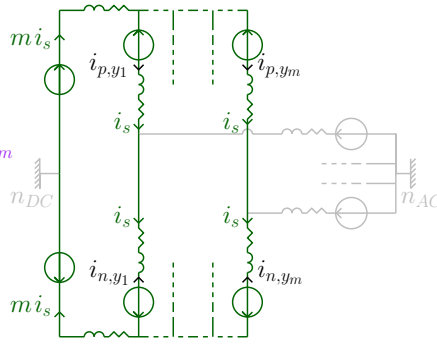

(b) Source Current

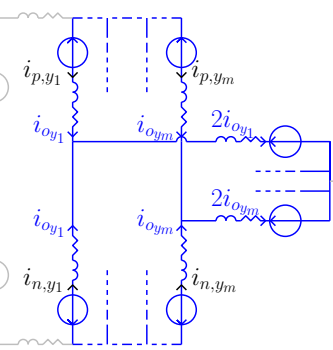

(d) Output Currents
Fig. 3. All four types of current flowing through the MMC

Substituting $i_{y_{j}}$ and $i_{y_{k}}$ in the first of those two expressions using (12) leads to:

$(m-1) i_{y_{j}}-\sum_{k \neq j}^{m} i_{y_{k}}=(m-1) 2\left(i_{m}+i_{o_{y_{j}}}\right)-\sum_{k \neq j}^{m} 2\left(i_{m}+i_{o_{y_{k}}}\right)$

Since $i_{m}$ does not depend on the sum index, it can be moved outside of the summation. Note that $\sum_{k \neq j}^{m}$ only contains $m-1$ terms:

$$
\sum_{k \neq j}^{m} 2\left(i_{m}+i_{o_{y_{k}}}\right)=(m-1) 2 i_{m}+\sum_{k \neq j}^{m} 2 i_{o_{y_{k}}}
$$

Combining (15) and (14) gives:

$$
(m-1) i_{y_{j}}-\sum_{k \neq j}^{m} i_{y_{k}}=(m-1) 2 i_{o_{y_{j}}}-\sum_{k \neq j}^{m} 2 i_{o_{y_{k}}}
$$

As explained previously, the sum of the entire set of output currents is null since the electrical current decoupling induces it. Accordingly:

$$
\sum_{k=1}^{m} i_{o_{y_{k}}}=0 \Longleftrightarrow i_{o_{y_{j}}}=-\sum_{k \neq j}^{m} i_{o_{y_{k}}}
$$

Replacing $-\sum_{k \neq j}^{m} i_{o_{y_{k}}}$ from this equation in (16) using (17), results in:

$$
(m-1) i_{y_{j}}-\sum_{k \neq j}^{m} i_{y_{k}}=(m-1) 2 i_{o_{j}}+2 i_{o_{y_{j}}}=2 m i_{o_{y_{j}}}
$$

Moving $-i_{y_{j}}$ within the sum reworks the previous equation into:

$$
m i_{y_{j}}-\sum_{k=1}^{m} i_{y_{k}}=2 m i_{o_{y_{j}}}
$$


Now subsituting $i_{y_{j}}$ thanks to (11) and using the contribution of (9) gives:

$$
m\left(i_{p, y_{j}}+i_{n, y_{j}}\right)-\left(i_{p}+i_{n}\right)=2 m i_{o_{y_{j}}}
$$

Therefore, it is concluded that:

$$
\forall y \in\left\{y_{1}, \ldots, y_{m}\right\}, \quad m\left(i_{p, y}+i_{n, y}\right)-\left(i_{p}+i_{n}\right)=2 m i_{o_{y}}
$$

This equation applies to any output current $i_{o_{y}}$.

The same approach can be followed for the circulating current. Substituting $i_{p, y_{j}}-i_{n, y_{j}}$ and $i_{p, y_{k}}-i_{n, y_{k}}$ in the second expression of (13) using (6) leads to:

$$
\begin{aligned}
& (m-1)\left(i_{p, y_{j}}-i_{n, y_{j}}\right)-\sum_{k \neq j}^{m}\left(i_{p, y_{k}}-i_{n, y_{k}}\right)= \\
& (m-1) 2\left(i_{s}+i_{c_{y_{j}}}\right)-\sum_{k \neq j}^{m} 2\left(i_{s}+i_{c_{y_{k}}}\right)
\end{aligned}
$$

Since $i_{s}$ does not depend on the index of the summation, the term $(m-1) 2 i_{s}$ is moved out of the sum, giving:

$(m-1) 2\left(i_{s}+i_{c_{y_{j}}}\right)-\sum_{k \neq j}^{m} 2\left(i_{s}+i_{c_{y_{k}}}\right)=(m-1) 2 i_{c_{y_{j}}}-\sum_{k \neq j}^{m} 2 i_{c_{y_{k}}}$

Since the sum of the entire set of circulating currents is null, it follows that:

$$
\sum_{k=1}^{m} i_{c_{y_{k}}}=0 \Longleftrightarrow i_{c_{y_{j}}}=-\sum_{k \neq j}^{m} i_{c_{y_{k}}}
$$

Substituting $-\sum_{k \neq j}^{m} i_{c_{y_{k}}}$ from (24) in (23), results in:

$$
\begin{aligned}
& (m-1) 2\left(i_{s}+i_{c_{y_{j}}}\right)-\sum_{k \neq j}^{m} 2\left(i_{s}+i_{c_{y_{k}}}\right)= \\
& (m-1) 2 i_{c_{y_{j}}}+2 i_{c_{y_{j}}}=2 m i_{c_{y_{j}}}
\end{aligned}
$$

Combining (22) and (25) yields:

$$
(m-1)\left(i_{p, y_{j}}-i_{n, y_{j}}\right)-\sum_{k \neq j}^{m}\left(i_{p, y_{k}}-i_{n, y_{k}}\right)=2 m i_{c_{y_{j}}}
$$

Moving $-\left(i_{p, y_{j}}-i_{n, y_{j}}\right)$ in the previous equation results in:

$$
m\left(i_{p, y_{j}}-i_{n, y_{j}}\right)-\sum_{k=1}^{m}\left(i_{p, y_{k}}-i_{n, y_{k}}\right)=2 m i_{c_{y_{j}}}
$$

From the contribution of (10) in (27), one finds:

$$
m\left(i_{p, y_{j}}-i_{n, y_{j}}\right)-\left(i_{p}-i_{n}\right)=2 m i_{c_{y_{j}}}
$$

Consequently, the conclusion is that:

$$
\forall y \in\left\{y_{1}, \ldots, y_{m}\right\}, \quad m\left(i_{p, y}-i_{n, y}\right)-\left(i_{p}-i_{n}\right)=2 m i_{c_{y}}
$$

Finally, the process followed in this part of the paper produced four useful equations, one for each type of current, that will help deriving the decoupled dynamics of all four currents independently. These useful equations are (9), (10), (21) and (29). To facilitate the forthcoming derivations, these equations are copied below. Thus, $\forall y \in\left\{y_{1}, \ldots, y_{m}\right\}$ :

$$
\begin{aligned}
& 2 m i_{m}=i_{p}+i_{n}=\sum_{y=y_{1}}^{y_{m}}\left(i_{p, y}+i_{n, y}\right) \\
& 2 m i_{s}=i_{p}-i_{n}=\sum_{y=y_{1}}^{y_{m}}\left(i_{p, y}-i_{n, y}\right) \\
& 2 m i_{o_{y}}=m\left(i_{p, y}+i_{n, y}\right)-\left(i_{p}+i_{n}\right) \\
& 2 m i_{c_{y}}=m\left(i_{p, y}-i_{n, y}\right)-\left(i_{p}-i_{n}\right)
\end{aligned}
$$

So as to derive the dynamic behavior of each type of current one can rely on those four equations. Note that, in (1) (or $\left(\alpha_{p, y}\right)$ ), the arm current $i_{p, y}$ appears similarly to $i_{n, y}$ in (2) (or $\left.\left(\alpha_{n, y}\right)\right)^{2}$. Accordingly, (30) suggests that in order to extract the dynamics of the common mode current $i_{m}$, the formula $\sum_{y=y_{1}}^{y_{m}}\left(\left(\alpha_{p, y}\right)+\left(\alpha_{n, y}\right)\right)$ should be developed. Following the same logic, (31) suggests that the dynamics of the DC source current $i_{s}$ will be found from $\sum_{y=y_{1}}^{y_{m}}\left(\left(\alpha_{p, y}\right)-\left(\alpha_{n, y}\right)\right)$. For the two remaining current types, the reasoning will lead to more complex formulas: $m\left(\left(\alpha_{p, y}\right)+\left(\alpha_{n, y}\right)\right)-\sum_{y=y_{1}}^{y_{m}}\left(\left(\alpha_{p, y}\right)+\left(\alpha_{n, y}\right)\right)$ for the output current $i_{o_{y}}$ behavior according to (32) and $m\left(\left(\alpha_{p, y}\right)-\left(\alpha_{n, y}\right)\right)-\sum_{y=y_{1}}^{y_{m}}\left(\left(\alpha_{p, y}\right)-\left(\alpha_{n, y}\right)\right)$ for the circulating current $i_{c_{y}}$ according to (33).

\section{Decoupled Current Dynamics}

1) Common Mode Current: For the common mode current $i_{m}$, the objective is to expand $\sum_{y=y_{1}}^{y_{m}}\left(\left(\alpha_{p, y}\right)+\left(\alpha_{n, y}\right)\right)$. In a first step, using (3) or (1) and (2) gives the following expression for $\left(\alpha_{p, y}\right)+\left(\alpha_{n, y}\right)$ :

$$
\begin{aligned}
v_{n}+v_{p}= & 2 v_{y}+\left[\left(R+2 R_{o}\right)+\left(L+2 L_{o}\right) s\right]\left(i_{p, y}+i_{n, y}\right) \\
& +\left(R_{s}+L_{s} s\right)\left(i_{p}+i_{n}\right)+2 v_{n_{A D}}+\left(v_{p, y}+v_{n, y}\right)
\end{aligned}
$$

Summing (34) on the entire set of phases gives $\sum_{y=y_{1}}^{y_{m}}\left(\left(\alpha_{p, y}\right)+\left(\alpha_{n, y}\right)\right)$ :

$$
\begin{aligned}
m\left(v_{n}+v_{p}\right) & =2 \sum_{y=y_{1}}^{y_{m}} v_{y} \\
& +\left[\left(R+2 R_{o}\right)+\left(L+2 L_{o}\right) s\right]\left(i_{p}+i_{n}\right) \\
& +m\left(R_{s}+L_{s} s\right)\left(i_{p}+i_{n}\right) \\
& +2 m v_{n_{A D}}+\sum_{y=y_{1}}^{y_{m}}\left(v_{p, y}+v_{n, y}\right)
\end{aligned}
$$

From (30), $i_{p}+i_{n}=2 m i_{m}$. Substituting $i_{p}+i_{n}$ in (35) and dividing by $2 m$ gives:

$$
\begin{aligned}
\frac{\left(v_{n}+v_{p}\right)}{2}= & \frac{1}{m} \sum_{y=y_{1}}^{y_{m}} v_{y} \\
& +\left[\left(m R_{s}+R+2 R_{o}\right)+\left(m L_{s}+L+2 L_{o}\right) s\right] i_{m} \\
& +v_{n_{A D}}+\frac{1}{2 m} \sum_{y=y_{1}}^{y_{m}}\left(v_{p, y}+v_{n, y}\right)
\end{aligned}
$$

As $i_{m}$ is the only current appearing in this equation, (36) is the differential equation governing the common mode current. A first decoupled differential equation was obtained. To simplify the equation, the following definitions are introduced:

$$
\left\{\begin{array}{l}
R_{m}^{e q}=m R_{s}+R+2 R_{o} \text { and } L_{m}^{e q}=m L_{s}+L+2 L_{o} \\
N_{\Sigma}(m)=\frac{1}{m}[1, \ldots, 1] \in \mathcal{M}_{1, m}(\mathbb{R}) \\
\frac{I_{m}}{V_{x}}=\left[i_{m}\right] \\
\frac{V_{y}}{V_{y}}=\left[v_{y_{1}}, \ldots, v_{n}\right]^{T} \\
\left.\frac{V_{n_{A D}}}{V_{p, y}}=\left[v_{n_{A D}}\right]=\left[v_{p, y_{1}}, \ldots, v_{n_{A C}}\right]_{m}\right]^{T} \\
\underline{V_{n, y}}=\left[v_{n, y_{1}}, \ldots, v_{n, y_{m}}\right]^{T}
\end{array}\right.
$$

Using these definitions, (36) becomes:

$$
\begin{aligned}
& \left(R_{m}^{e q}+L_{m}^{e q} s\right) \underline{I_{m}}=-\frac{1}{2} N_{\Sigma}(m)\left(\underline{V_{p, y}}+\underline{V_{n, y}}\right) \\
& +\left(N_{\Sigma}(2) \underline{V_{x}}-N_{\Sigma}(m) \underline{V_{y}}-N_{\Sigma}(1) \underline{V_{n_{A D}}}\right)
\end{aligned}
$$

\footnotetext{
${ }^{2}$ Equation (1) is also named $\left(\alpha_{p, y}\right)$ and (2) is also named $\left(\alpha_{n, y}\right)$, see (3)
} 
The color of this equation and the upcoming ones correspond to those of Fig. 3. In the case where the DC and AC neutrals are disconnected, the common mode current is structurally forced to zero, transforming the previous equation into:

$$
\underline{V_{n_{A D}}}=-\frac{1}{2} N_{\Sigma}(m)\left(\underline{V_{p, y}}+\underline{V_{n, y}}\right)+N_{\Sigma}(2) \underline{V_{x}}-N_{\Sigma}(m) \underline{V_{y}}
$$

2) Source Current (DC-side): For the source current $i_{s}$, the objective is to expand $\sum_{y=y_{1}}^{y_{m}}\left(\left(\alpha_{p, y}\right)-\left(\alpha_{n, y}\right)\right)$. First of all, using (3) or (1) and (2) gives the following equation for $\left(\alpha_{p, y}\right)-\left(\alpha_{n, y}\right)$ :

$$
\begin{aligned}
v_{p}-v_{n}= & (R+L s)\left(i_{p, y}-i_{n, y}\right) \\
& +\left(R_{s}+L_{s} s\right)\left(i_{p}-i_{n}\right)+\left(v_{p, y}-v_{n, y}\right)
\end{aligned}
$$

Summing (40) on the entire set of phases yields $\sum_{y=y_{1}}^{y_{m}}\left(\left(\alpha_{p, y}\right)-\left(\alpha_{n, y}\right)\right)$ :

$$
\begin{aligned}
& m\left(v_{p}-v_{n}\right)=(R+L s)\left(i_{p}-i_{n}\right) \\
& +m\left(R_{s}+L_{s} s\right)\left(i_{p}-i_{n}\right)+\sum_{y=y_{1}}^{y_{m}}\left(v_{p, y}-v_{n, y}\right)
\end{aligned}
$$

From (31), $i_{p}-i_{n}=2 m i_{s}$. Substituting $i_{p}-i_{n}$ in (41) and dividing by $2 m$ implies that:

$$
\begin{aligned}
\frac{\left(v_{p}-v_{n}\right)}{2}= & {\left[\left(m R_{s}+R\right)+\left(m L_{s}+L\right) s\right] i_{s} } \\
& +\frac{1}{2 m} \sum_{y=y_{1}}^{y_{m}}\left(v_{p, y}-v_{n, y}\right)
\end{aligned}
$$

A single type of current $\left(i_{s}\right)$ appears in equation (42), so that the second decoupled differential equation has been obtained. As was done previously for the common mode current, specific notations are introduced in order to simplify the equation:

$$
\left\{\begin{array}{l}
R_{s}^{e q}=m R_{s}+R \text { and } L_{s}^{e q}=m L_{s}+L \\
N_{\Delta}(m)=\frac{1}{m}\left[m \mathbb{I}_{m}-\mathbb{J}_{m}\right] \in \mathcal{M}_{m}(\mathbb{R}) \\
\underline{I_{s}}=\left[i_{s}\right]
\end{array}\right.
$$

Where $\mathbb{I}_{m}$ is the identity matrix of size $m$ and $\mathbb{J}_{a, b}$ is the $(a \times b)$ matrix filled with $1^{\prime} s$, which means that $\mathbb{J}_{m}=\mathbb{J}_{m, m}$. Using the definitions, (42) becomes:

$$
\begin{aligned}
\left(R_{s}^{e q}+L_{s}^{e q} s\right) \underline{I_{s}}= & -\frac{1}{2} N_{\Sigma}(m)\left(\underline{V_{p, y}-} V_{n, y}\right) \\
& +\left(\left[\begin{array}{ll}
1 & 0
\end{array}\right] N_{\Delta}(2) \underline{V_{x}}\right)
\end{aligned}
$$

3) Circulating Current: For the circulating current, the same reasoning as the one applied for the common mode current and the source current will be applied. However, the procedure will be slightly more complex. For a given circulating current $i_{c_{y}}$, the objective is to transform $m\left(\left(\alpha_{p, y}\right)-\left(\alpha_{n, y}\right)\right)-\sum_{y=y_{1}}^{y_{m}}\left(\left(\alpha_{p, y}\right)-\left(\alpha_{n, y}\right)\right)$. Since $\sum_{y=y_{1}}^{y_{m}}\left(\left(\alpha_{p, y}\right)-\left(\alpha_{n, y}\right)\right)$ was already developed in (41), $m\left(\left(\alpha_{p, y}\right)-\left(\alpha_{n, y}\right)\right)$ is expressed using (1) and (2), which yields the following equation for $m\left(\left(\alpha_{p, y}\right)-\left(\alpha_{n, y}\right)\right)$ :

$$
\begin{aligned}
& m\left(v_{p}-v_{n}\right)=m(R+L s)\left(i_{p, y}-i_{n, y}\right) \\
& \quad+m\left(R_{s}+L_{s} s\right)\left(i_{p}-i_{n}\right)+m\left(v_{p, y}-v_{n, y}\right)
\end{aligned}
$$

Subtracting $\sum_{y=y_{1}}^{y_{m}}\left(\left(\alpha_{p, y}\right)-\left(\alpha_{n, y}\right)\right) \quad$ in (41) from $m\left(\left(\alpha_{p, y}\right)-\left(\alpha_{n, y}\right)\right)$ in (45) gives $m\left(\left(\alpha_{p, y}\right)-\left(\alpha_{n, y}\right)\right)$ $\sum_{y=y_{1}}^{y_{m}}\left(\left(\alpha_{p, y}\right)-\left(\alpha_{n, y}\right)\right)$ :

$$
\begin{aligned}
0= & (R+L s)\left(m\left(i_{p, y}-i_{n, y}\right)-\left(i_{p}-i_{n}\right)\right) \\
& +\left(m\left(v_{p, y}-v_{n, y}\right)-\sum_{y=y_{1}}^{y_{m}}\left(v_{p, y}-v_{n, y}\right)\right)
\end{aligned}
$$

From (33), $m\left(i_{p, y}-i_{n, y}\right)-\left(i_{p}-i_{n}\right)=2 m i_{c_{y}}$. Substituting $m\left(i_{p, y}-i_{n, y}\right)-\left(i_{p}-i_{n}\right)$ in (46) and dividing by $2 m$ yields:

$$
\begin{aligned}
0= & (R+L s) i_{c_{y}} \\
& +\frac{1}{2 m}\left(m\left(v_{p, y}-v_{n, y}\right)-\sum_{y=y_{1}}^{y_{m}}\left(v_{p, y}-v_{n, y}\right)\right)
\end{aligned}
$$

As was done previously, notations are introduced to simplify equation (47):

$$
\left\{\begin{array}{l}
R_{c}^{e q}=R \text { and } L_{c}^{e q}=L \\
\underline{I_{c}}=\left[i_{c_{1}}, \ldots, i_{c_{m}}\right]^{T}
\end{array}\right.
$$

Since (47) is true for any leg (i.e., any y), the following equation encapsulates the dynamics of all circulating currents into a single vector differential equation with $m$ elements:

$$
\left(R_{c}^{e q}+L_{c}^{e q} s\right) \underline{I_{c}}=-\frac{1}{2} N_{\Delta}(m)\left(\underline{V_{p, y}}-\underline{V_{n, y}}\right)
$$

4) Output Current (AC-side): For the output current, the same procedure used for the circulating current is applied. For a given output current $i_{o_{y}}$, the objective is to develop $m\left(\left(\alpha_{p, y}\right)+\left(\alpha_{n, y}\right)\right)-\sum_{y=y_{1}}^{y_{m}}\left(\left(\alpha_{p, y}\right)+\left(\alpha_{n, y}\right)\right)$. Since $\sum_{y=y_{1}}^{y_{m}}\left(\left(\alpha_{p, y}\right)+\left(\alpha_{n, y}\right)\right)$ was already expanded in (35), $m\left(\left(\alpha_{p, y}\right)+\left(\alpha_{n, y}\right)\right)$ is expressed using (1) and (2):

$$
\begin{aligned}
& m\left(v_{p}+v_{n}\right)=2 m v_{y} \\
& \quad+m\left[\left(R+2 R_{o}\right)+\left(L+2 L_{o}\right) s\right]\left(i_{p, y}+i_{n, y}\right) \\
& \quad+m\left(R_{s}+L_{s} s\right)\left(i_{p}+i_{n}\right) \\
& \quad+2 m v_{n_{A D}}+m\left(v_{p, y}+v_{n, y}\right)
\end{aligned}
$$

Subtracting $\sum_{y=y_{1}}^{y_{m}}\left(\left(\alpha_{p, y}\right)+\left(\alpha_{n, y}\right)\right) \quad$ in (35) from $m\left(\left(\alpha_{p, y}\right)+\left(\alpha_{n, y}\right)\right)$ in (50) gives $m\left(\left(\alpha_{p, y}\right)+\left(\alpha_{n, y}\right)\right)-$ $\sum_{y=y_{1}}^{y_{m}}\left(\left(\alpha_{p, y}\right)+\left(\alpha_{n, y}\right)\right)$ :

$$
\begin{aligned}
0= & 2\left(m v_{y}-\sum_{y=y_{1}}^{y_{m}} v_{y}\right) \\
& +\left[\left(R+2 R_{o}\right)+\left(L+2 L_{o}\right) s\right]\left(m\left(i_{p, y}+i_{n, y}\right)-\left(i_{p}+i_{n}\right)\right) \\
& +\left(m\left(v_{p, y}+v_{n, y}\right)-\sum_{y=y_{1}}^{y_{m}}\left(v_{p, y}+v_{n, y}\right)\right)
\end{aligned}
$$

From (32), $\left(m\left(i_{p, y}+i_{n, y}\right)-\left(i_{p}+i_{n}\right)\right)=2 m i_{o_{y}}$. Substituting $\left(m\left(i_{p, y}+i_{n, y}\right)-\left(i_{p}+i_{n}\right)\right)$ in (51) and dividing by $2 m$ gives:

$$
\begin{gathered}
-\frac{1}{m}\left(m v_{y}-\sum_{y=y_{1}}^{y_{m}} v_{y}\right)=\left[\left(R+2 R_{o}\right)+\left(L+2 L_{o}\right) s\right] i_{o_{y}} \\
+\frac{1}{2 m}\left(m\left(v_{p, y}+v_{n, y}\right)-\sum_{y=y_{1}}^{y_{m}}\left(v_{p, y}+v_{n, y}\right)\right)
\end{gathered}
$$

The following notation is introduced to simplify equation (52):

$$
\left\{\begin{array}{l}
R_{o}^{e q}=R+2 R_{o} \text { and } L_{o}^{e q}=L+2 L_{o} \\
\underline{I_{o}}=\left[i_{o_{1}}, \ldots, i_{o_{m}}\right]^{T}
\end{array}\right.
$$

Since (52) is true for any leg (i.e., any y), the following equation encapsulates the dynamics of all output currents into a single vector differential equation with $m$ elements:

$$
\begin{aligned}
\left(R_{o}^{e q}+L_{o}^{e q} s\right) \underline{I_{o}}= & -\frac{1}{2} N_{\Delta}(m)\left(\underline{V_{p, y}}+\underline{V_{n, y}}\right) \\
& +\left(-N_{\Delta}(m) \underline{V_{y}}\right)
\end{aligned}
$$


At this point, all four types of current have been shown to satisfy independent differential equations driven by the MMC arm voltages. Thus, the currents dynamics are decoupled by current type and are governed by (38) for the common mode current, by (44) for the DC source current, by (49) for the circulating currents and by (54) for the AC output currents.

\section{Full-Order Current State-Space Model}

The state variables of the model are the components of the currents, so the state vector is:

$$
\underline{X_{H L}}=\left[\underline{I_{m}}, \underline{I_{s}}, \underline{I_{c}^{T}}, \underline{I_{o}^{T}}\right]^{T}
$$

Equations (38) and (44) both represent the behavior of a single current while (49) and (54) describe the dynamics of $m$ different currents, with a total of $2 m+2$ currents. The state vector $X_{H L}$ contains all these currents, so the order of the state-space model is $2 m+2$ and varies according to the number of phases of the AC network.

The state-space model is given by the following state equation:

$$
\left\{\begin{array}{l}
\dot{X}_{H L}=A_{H L} \underline{X_{H L}}+B_{H L} \underline{U_{H L}}+\underline{E_{H L}} \\
\underline{Y_{H L}}=C_{H L} \underline{X_{H L}}+D_{H L} \underline{U_{H L}}
\end{array}\right.
$$

where:

$$
\begin{gathered}
A_{H L}=\left[\begin{array}{ccc}
-\frac{R_{m}^{e q}}{L_{m}^{e q}} & & \\
& -\frac{R_{s}^{e q}}{L_{s}^{e q}} & \\
& -\frac{R_{c}^{e q}}{L_{c}^{e q}} \mathbb{I}_{m} & \\
& & -\frac{R_{e}^{e q}}{L_{o}^{e q}} \mathbb{I}_{m}
\end{array}\right] \in \mathcal{M}_{2 m+2}(\mathbb{R}) \\
B_{H L}=-\frac{1}{2}\left[\begin{array}{ll}
N_{\Sigma}(m) / L_{m}^{e q} & N_{\Sigma}(m) / L_{m}^{e q} \\
N_{\Sigma}(m) / L_{s}^{e q} & -N_{\Sigma}(m) / L_{s}^{e q} \\
N_{\Delta}(m) / L_{c}^{e q} & -N_{\Delta}(m) / L_{c}^{e q} \\
N_{\Delta}(m) / L_{o}^{e q} & N_{\Delta}(m) / L_{o}^{e q}
\end{array}\right] \in \mathcal{M}_{2 m+2,2 m}(\mathbb{R})
\end{gathered}
$$

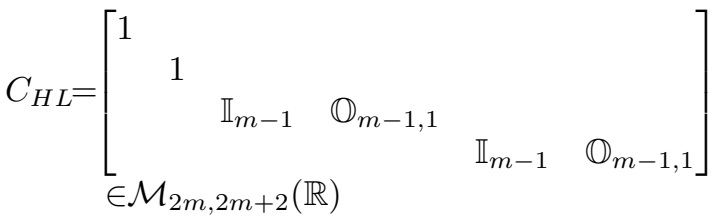$$
D_{H L}=\left[\mathbb{O}_{2 m, 2 m}\right] \in \mathcal{M}_{2 m}(\mathbb{R})
$$$$
\underline{U_{H L}}=\left[v_{p, y_{1}}, \ldots, v_{p, y_{m}}, v_{n, y_{1}}, \ldots, v_{n, y_{m}}\right]^{T} \in \mathcal{M}_{2 m, 1}(\mathbb{R})
$$

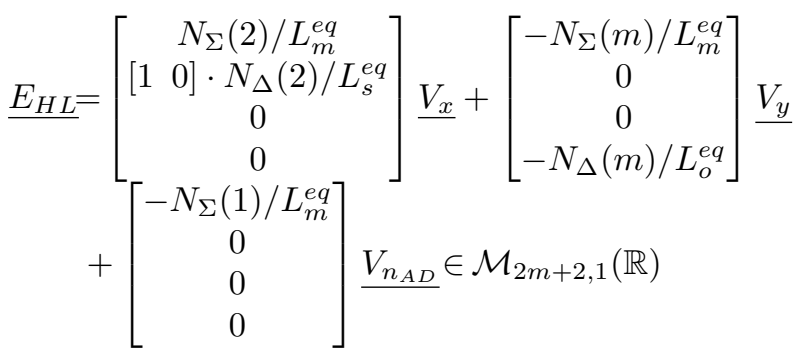

and $\mathbb{O}_{a, b}$ is the $(a \times b)$ null matrix. As the sum of the circulating currents and the sum of the output currents are equal to zero, one of the currents is redundant. The same is true for the output currents. The choice was made to drop the last circulating current $i_{c_{m}}$ out of the output vector $Y_{H L}$, and the last output current $i_{o_{m}}$. Hence the form taken by $C_{H L}$.

The control variables are the arm voltages $v_{x, y}$. It is readily possible to adapt equations (55)-(57) to the number $m$ of phases and the equations are independent of $N$. All together, they embody the General Polyphased Full Order Current State-Space Model (GPFOCSSM).

\section{Model Simulation And Testing}

\section{A. Test Procedure}

To ensure that the model developed here properly represents the behavior of the MMC currents, a verification procedure is set up using MATLAB $\AA$. The state-space model is coded in Simulink ${ }^{\circledR}$ using a Matlab-Function able to adapt itself depending on the number of MMC legs and neutral points connection, as shown in Fig. 4. In parallel, the electrical diagram of Fig. 1 is also simulated with the PLECS®-Blockset in Simulink®, as shown in Fig. 5.

The model as well as the electrical circuit are simulated jointly, and the results are compared as shown on Fig. 6. In the accuracy tests to follow, the plots will show the four types of currents, including those computed by the state-space model as well as those computed by the circuit simulator. Finally, the plots will highlight the difference between the two sets of electrical signals.

To verify the model, the most general operating case is considered. This case is characterized by an active AC grid, the AC and DC neutrals connected and non-zero control signals. This is implemented by: $\forall y \in\left\{y_{1}, \ldots, y_{m}\right\}, v_{y}=$ $\hat{V}_{A C} \sin \left(\omega_{o} t-\varphi_{y}\right)$ with $\varphi_{y_{k}}=(k-1) 2 \pi / m$ for having an active AC grid; by an electrical link in the PLECS $®$ diagram between the AC and DC neutrals to connect them ${ }^{3}$; and by $\forall x \in\{p, n\}, \forall y \in\left\{y_{1}, \ldots, y_{m}\right\}, v_{x, y} \neq 0$. The question then arises as the choice of non-zero control signals $v_{x, y}$. In a first approach, the currents are not controlled and the

${ }^{3}$ Connecting neutrals implies that $v_{n_{A C}}=v_{n_{D C}}$, thus $V_{n_{A D}}=0$ for the model and the circuit simulator, therefore $\epsilon_{V_{n}}=0$. This error won't be shown in the upcoming curves, thus 4 out of 5 error curves are presented.

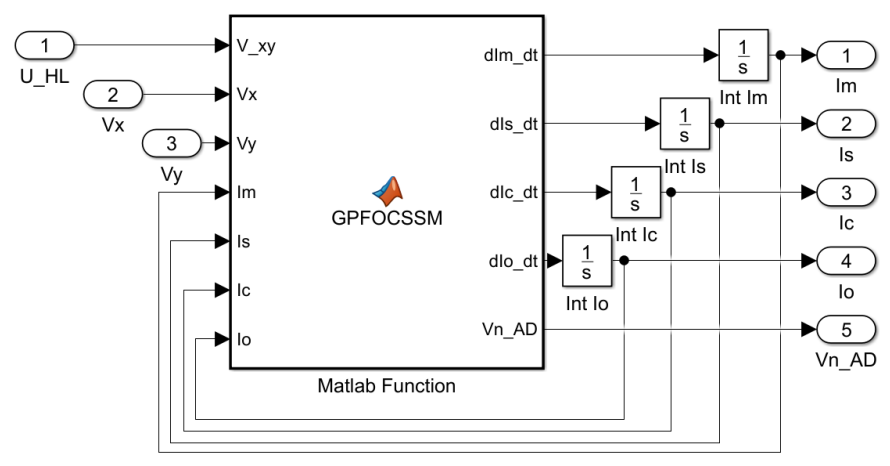

Fig. 4. State-space model Matlab Function used in Simulink® 


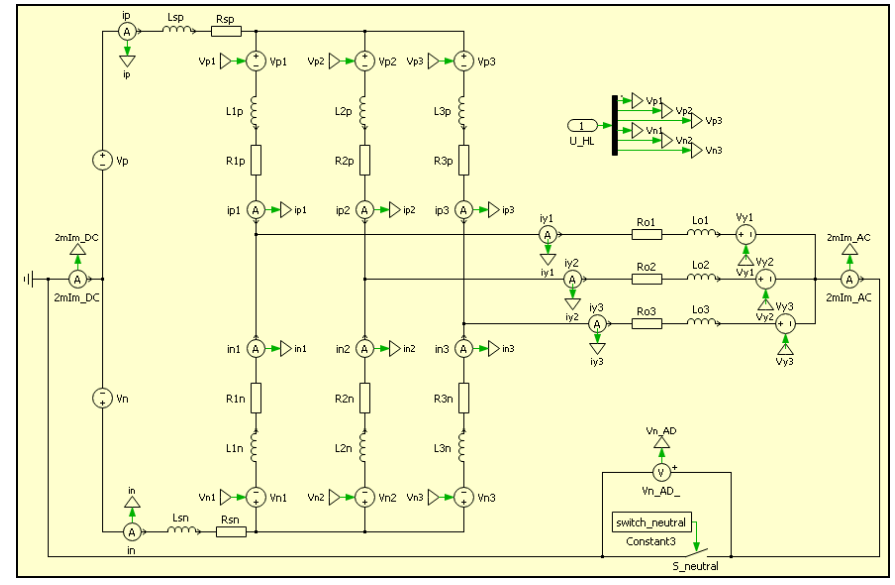

Fig. 5. Electrical circuit built with PLECS $®$ Blockset - here for $m=3$

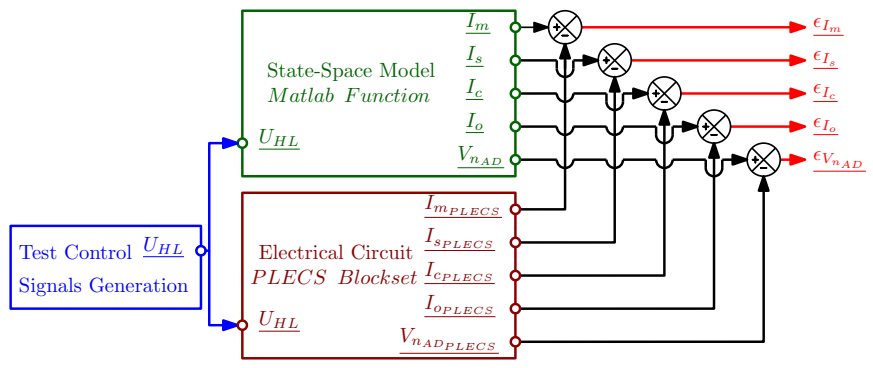

Fig. 6. Validation principle of the state-space model in Simulink®

direct modulation method is used (as in [15], among others). Accordingly:

$\forall y \in\left\{y_{1}, \ldots, y_{m}\right\},\left\{\begin{array}{l}v_{p, y}=\frac{V_{D C}}{2}\left[1-M \cos \left(\omega_{o} t-\varphi_{y}\right)\right] \\ v_{n, y}=-\frac{V_{D C}}{2}\left[1+M \cos \left(\omega_{o} t-\varphi_{y}\right)\right]\end{array}\right.$

The the modulation index $M$ is set to 1 . The choice of such control signals is justified by the fact that they make it possible to sweep the entire voltage range that can be reached by the MMC arms. However, using directly those control signals won't allow all four types of currents to evolve over time. For example, (36) implies that the common mode current is governed by $\sum_{y=y_{1}}^{y_{m}}\left(v_{p, y}+v_{n, y}\right)$, and according to the choice made in (58), $v_{p, y}+v_{n, y}=-V_{D C} M \cos \left(\omega_{o} t-(k-1) 2 \pi / m\right)$. Generally, $\sum_{y=y_{1}}^{y_{m}} \cos \left(\omega_{o} t-(k-1) 2 \pi / m\right)=0$, and therefore $\sum_{y=y_{1}}^{y_{m}}\left(v_{p, y}+v_{n, y}\right)=0$. In other words, the control signals do not influence the common mode current. Other control signals are therefore necessary to fully test the model. A slight update in the definitions of (58) will enable this feature for all curents:

$\forall y \in\left\{y_{1}, \ldots, y_{m}\right\},\left\{\begin{array}{l}v_{p, y}=\frac{V_{D C}}{2}\left[1-M \cos \left(\omega_{o} t-\varphi_{y}\right)\right] \\ v_{n, y}=-\frac{V_{D C}}{2}\left[\frac{1}{2}+\frac{M}{2} \cos \left(\omega_{o} t-\varphi_{y}\right)\right]\end{array}\right.$

\section{B. Accuracy Assessment of the Model}

Simulations were carried out for several operating cases and, in particular, for several values of the number of $m$ of phases. To highlight the interest of the model developed here, the curves obtained for the case where $m=7$ are shown. The
TABLE I

MMC SIMULATION PARAMETERS

\begin{tabular}{|c|c|c|}
\hline Meaning & Symbol & Value \\
\hline Bus voltage & $V_{D C}$ & $600 \mathrm{~V}$ \\
Half-bus voltage & $v_{p}=-v_{n}$ & $V_{D C} / 2=300 \mathrm{~V}$ \\
Bus resistance and inductance & $R_{s}, L_{s}$ & $50 \mathrm{~m} \Omega, 2 \mathrm{mH}$ \\
\hline Switching frequency and period & $f_{s}, T_{s}$ & $2 \mathrm{kHz}, 500 \mu \mathrm{s}$ \\
Arm resistance and inductance & $R, L$ & $10 \mathrm{~m} \Omega, 5 \mathrm{mH}$ \\
\hline AC active voltage & $\hat{V}_{A C}$ & $230 \cdot \sqrt{2} \simeq 325 \mathrm{~V}$ \\
AC grid frequency and period & $f_{o}, T_{o}$ & $50 \mathrm{~Hz}, 20 \mathrm{~ms}$ \\
AC grid pulsation & $\omega_{o}$ & $2 \pi f_{o} \simeq 314 \mathrm{rad} / \mathrm{s}$ \\
AC load resistance and inductance & $R_{o}, L_{o}$ & $40 \Omega, 5 \mathrm{mH}$ \\
\hline Simulation time step & $T_{\text {step }}$ & $T_{s} / 50=10 \mu \mathrm{s}$ \\
Simulation end time & $t_{\text {end }}$ & $7 \cdot T_{o}=140 \mathrm{~ms}$ \\
\hline
\end{tabular}

parameters of the MMC correspond to those of a converter available at the LAPLACE laboratory, part of which are given in [16]. The whole set is listed in Table I.

The control signals defined in (59) were applied in the simulation for seven phases, resulting in the following current curves. Note that important circulating currents appear, this is due to the unusual forms of $v_{p, y}$ and $v_{n, y}$ using different modulation indexes.

For each type of current, the bottom plot shows the difference between the current from the state-space model and the same current from PLECS $®$. Figs. 7, 8, 9, and 10 show that the maximum difference between GPFOCSSM and PLECS $®$ is on the circulating current $I_{c}$. The deviation is of the order of $2.07 \cdot 10^{-9} \mathrm{~A}$ which is very low compared to the magnitude of the current signals. This allows to validate the accuracy of the state-space model.

\section{Conclusions}

A state-space model of the MMC was derived to describe the dynamics of the currents with the arm voltages as input variables. A first feature of the model was the decomposition into the four independent current components for the general case of electrical polyphased systems. An important feature of the model was its modularity both in the number $m$ of phases and in the number $N$ of submodules. Also, the model was
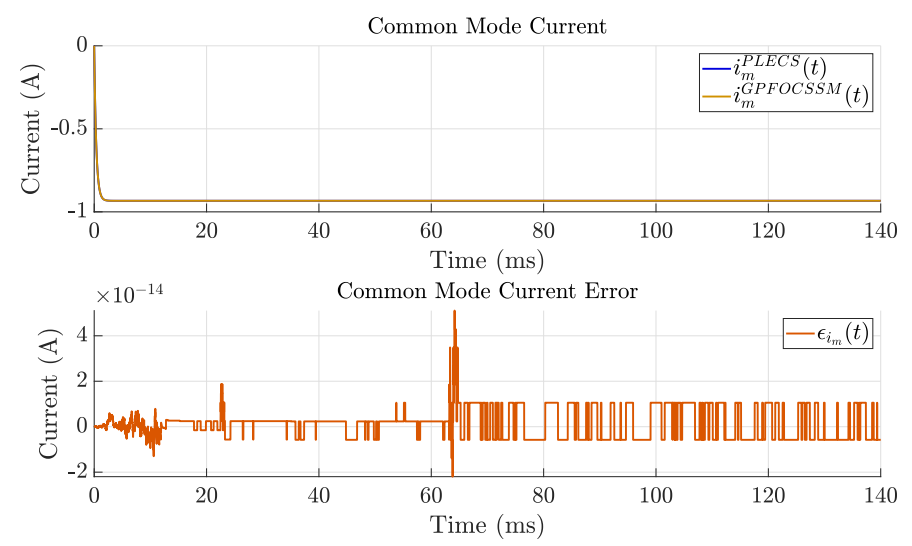

Fig. 7. Common mode current and difference with PLECS $®-m=7$ 

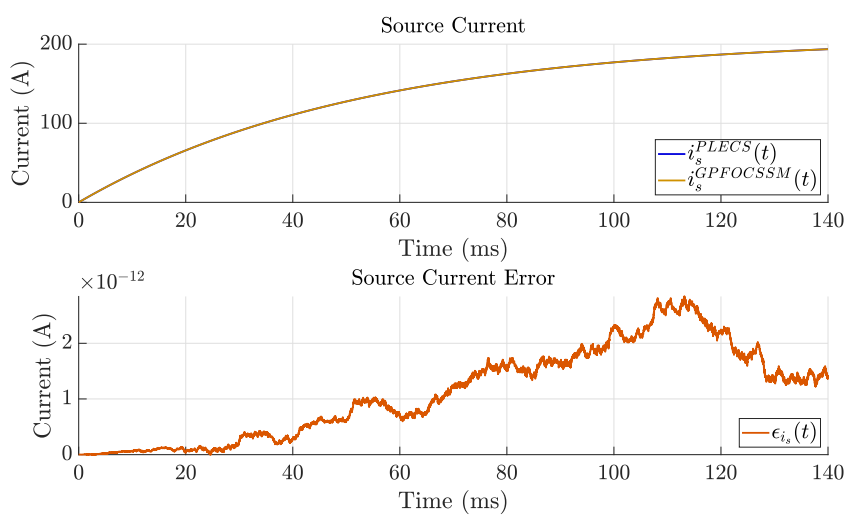

Fig. 8. Source currents and difference with PLECS $®-m=7$
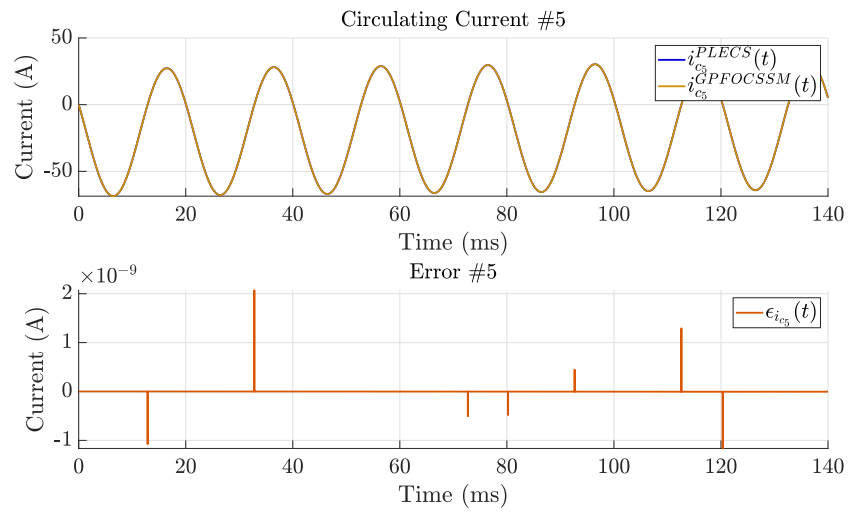

Fig. 9. Circulating current $\# 5$ out of 7 and difference PLECS $®-m=7$
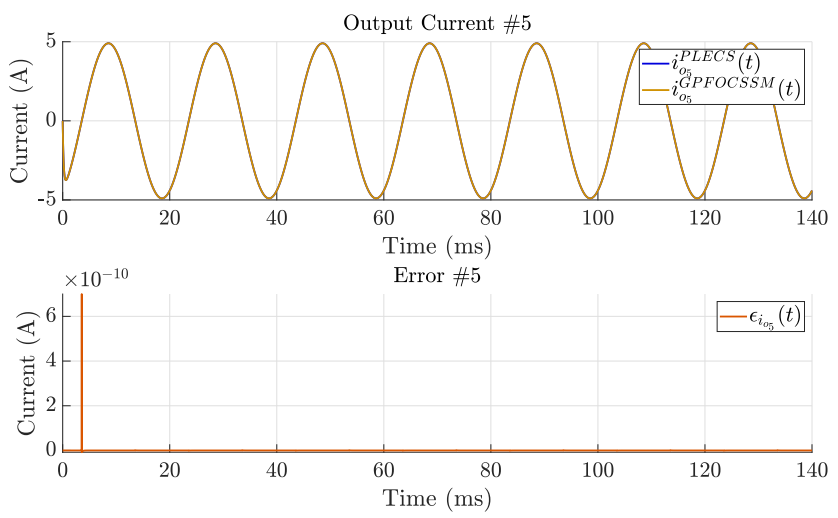

Fig. 10. Output current $\# 5$ out of 7 and difference PLECS $®-m=7$

developed to be adaptive to the active or passive nature of the AC-grid as well as to the connection between the AC and DC neutrals. The model was evaluated in simulation and the results were found consistent with a circuit-based simulation software, thus validating the state-space model's accuracy.

The model was derived using simplifying assumptions concerning the influence of the capacitors of each submodules. It could be interesting to remove these assumptions in order to obtain a more general model.

The first benefit of the developed model is that it enables the analysis of all four current types flowing throught an MMC connected to an $\mathrm{AC}$ grid having any given number of phases, for any type of neutral connection and any nature of the ACside grid. Thanks to this model it will be possible to design generic current control algorithms for MMCs. Thus to control a given MMC it will be sufficient to specify to the algorithm the parameters of the MMC, of the AC network and of the DC bus without having to undergo major changes in the control algorithm structure.

\section{REFERENCES}

[1] J. Arrillaga, Y. Liu, and N. Watson, "Flexible Power Transmission: The HVDC Options," Flexible Power Transmission: The HVDC Options, pp. 1-362, Sep. 2007.

[2] A. Lesnicar and R. Marquardt, "An innovative modular multilevel converter topology suitable for a wide power range," in 2003 IEEE Bologna Power Tech Conference Proceedings, vol. 3, Jun. 2003.

[3] K. Sharifabadi, L. Harnefors, H.-P. Nee, S. Norrga, and R. Teodorescu, "Introduction to modular multilevel converters," in Design, control, and application of modular multilevel converters for HVDC transmission systems. IEEE, 2016, pp. 7-59.

[4] M. Bodson, "Evaluation of optimization methods for control allocation," Journal of Guidance, Control, and Dynamics, vol. 25, no. 4, pp. 703711, Jul. 2002.

[5] R. Marquardt, "Modular multilevel converters: state of the art and future progress," IEEE Power Electronics Magazine, vol. 5, no. 4, pp. 24-31, Dec. 2018.

[6] K. Sharifabadi, L. Harnefors, H.-P. Nee, S. Norrga, and R. Teodorescu, "Dynamics and control," in Design, control, and application of modular multilevel converters for HVDC transmission systems. IEEE, 2016, pp. 133-213.

[7] J. Freytes, S. Akkari, J. Dai, F. Gruson, P. Rault, and X. Guillaud, "Small-signal state-space modeling of an HVDC link with modular multilevel converters," in 2016 IEEE 17th Workshop on Control and Modeling for Power Electronics (COMPEL). Trondheim, Norway: IEEE, Jun. 2016, pp. 1-8.

[8] G. Bergna-Diaz, J. Freytes, X. Guillaud, S. D'Arco, and J. A. Suul, "Generalized voltage-based state-space modeling of modular multilevel converters with constant equilibrium in steady state," IEEE Journal of Emerging and Selected Topics in Power Electronics, vol. 6, no. 2, pp. 707-725, Jun. 2018.

[9] S. Bacha, I. Munteanu, and A. I. Bratcu, Power Electronic Converters Modeling and Control: with Case Studies, ser. Advanced Textbooks in Control and Signal Processing. London: Springer-Verlag, 2014.

[10] R. Lizana, M. A. Perez, D. Arancibia, J. R. Espinoza, and J. Rodriguez, "Decoupled current model and control of modular multilevel converters," IEEE Transactions on Industrial Electronics, vol. 62, no. 9, pp. 53825392, Sep. 2015.

[11] A. Bouarfa, "Méthodes de commande par allocation de convertisseurs statiques polyphasés, multi-niveaux : de la modélisation à la mise en oeuvre temps-réel," Ph.D. Thesis, Université Toulouse III - PaulSabatier, Nov. 2017.

[12] M. Zama, "Modeling and control of modular multilevel converters (MMCs) for HVDC applications," Ph.D. Thesis, Communauté Université Grenoble Alpes, 2017.

[13] M. A. Perez, J. Rodriguez, and S. Bernet, "Decoupled capacitor voltage control of modular multilevel converters," in 2014 IEEE Energy Conversion Congress and Exposition (ECCE), Sep. 2014, pp. 4947-4953.

[14] D. Karwatzki and A. Mertens, "Generalized control approach for a class of modular multilevel converter topologies," IEEE Transactions on Power Electronics, vol. 33, no. 4, pp. 2888-2900, Apr. 2018, conference Name: IEEE Transactions on Power Electronics.

[15] D. Siemaszko, A. Antonopoulos, K. Ilves, M. Vasiladiotis, L. Ängquist, and H.-P. Nee, "Evaluation of control and modulation methods for modular multilevel converters," in The 2010 International Power Electronics Conference (ECCE ASIA), Jun. 2010, pp. 746-753.

[16] N. Serbia, "Modular multilevel converters for HVDC power stations," Ph.D. Thesis, Institut National Polytechnique de Toulouse, 2014. 